\title{
Intercomparison of erythemal broadband radiometers calibrated by seven UV calibration facilities in Europe and the USA
}

\author{
G. Hülsen ${ }^{1}$, J. Gröbner ${ }^{1}$, A. Bais ${ }^{2}$, M. Blumthaler ${ }^{3}$, P. Disterhoft ${ }^{4}$, B. Johnsen ${ }^{5}$, K. O. Lantz ${ }^{4}$, C. Meleti ${ }^{2}$, J. Schreder ${ }^{6}$, \\ J. M. Vilaplana Guerrero ${ }^{7}$, and L. Ylianttila ${ }^{8}$ \\ ${ }^{1}$ Physikalisch-Meteorologisches Observatorium Davos/World Radiation Center, Dorfstrasse 30, \\ 7278 Davos Dorf, Switzerland \\ ${ }^{2}$ Aristotle University of Thessaloniki, Laboratory of Atmospheric Physics, Campus Box 149, 54124 Thessaloniki, Greece \\ ${ }^{3}$ Innsbruck Medical University, Division for Biomedical Physics, Müllerstr. 44, 6020 Innsbruck, Austria \\ ${ }^{4}$ National Oceanic and Atmospheric Administration, Central UV Calibration Facility, 325 Broadway, \\ 80305 Boulder, CO, USA \\ ${ }^{5}$ Norwegian Radiation Protection Authority, Grini Naeringspark 13, 1361 Osteras, Norway \\ ${ }^{6}$ CMS Ing. Dr. Schreder GmbH, Eggerstrasse 8, 6322 Kirchbichl, Austria \\ ${ }^{7}$ Instituto Nacional de Técnica Aerospacial, Estación de Sondeos Atmosféricos El Arenosillo, 21130 Mazagon, Spain \\ ${ }^{8}$ STUK, Radiation and Nuclear Safety Authority, Non-Ionizing Radiation Laboratory, P.O. Box 14, 00881 Helsinki, Finland
}

Received: 7 December 2007 - Published in Atmos. Chem. Phys. Discuss.: 6 February 2008

Revised: 9 April 2008 - Accepted: 31 July 2008 - Published: 21 August 2008

\begin{abstract}
A bi-lateral intercomparison of erythemal broadband radiometers was performed between seven UV calibration facilities. The calibrations provided by the instruments owners were compared relative to the characterisation and calibration performed at PMOD/WRC in Davos, Switzerland. The calibration consisted in the determination of the spectral and angular response of the radiometer, followed by an absolute calibration performed outdoors relative to a spectroradiometer which provided the absolute reference.

The characterization of the detectors in the respective laboratories are in good agreement: The determinations of the angular responses have deviations below $\pm 4 \%$ and the spectral responses agree within $\pm 20 \%$. A "blind" intercomparison of the erythemally weighted irradiances derived by the respective institutes and PMOD/WRC showed consistent measurements to within $\pm 2 \%$ for the majority of institutes. One institute showed slightly larger deviation of $10 \%$. The differences found between the different instrument calibrations are all within the combined uncertainty of the calibration.
\end{abstract}

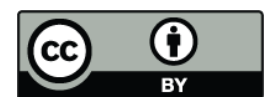

Correspondence to: G. Hülsen (gregor.huelsen@pmodwrc.ch)

\section{Introduction}

Routine measurements of solar ultraviolet (UV) radiation are often performed with UV broadband radiometers due to their simple operational requirements. Even though the operation of these radiometers is straightforward (they require only a power supply and a voltmeter), the relationship between the raw signal and the desired UV radiation product is complex and requires an elaborate characterization and calibration procedure for each individual broadband radiometer (Lantz et al., 1999; Leszczynski et al., 1998; Hülsen and Gröbner, 2007).

Here, we will compare the calibrations of six broadband radiometers performed by 6 UV calibration facilities (UVCF) in Europe and the United States with the calibration performed by the European reference UV calibration facility of the PMOD/WRC (see Table 1). This exercise was part of a large-scale intercomparison and calibration campaign organized within the COST726 activities and hosted by PMOD/WRC in August 2006 (Gröbner et al., 2007).

The comparisons were organized as "blind comparisons", i.e. the results were only communicated to the participants at the end of the measurement campaign when all data were delivered to PMOD/WRC. The calibration comparison results will be presented as bi-lateral comparisons between the owner's institute and PMOD/WRC and therefore allow a

Published by Copernicus Publications on behalf of the European Geosciences Union. 
Table 1. UV calibration facilities participating in the intercomparison.

\begin{tabular}{|c|c|c|c|}
\hline UV calibration facility & Country & Abbreviation & Instrument \\
\hline Physikalisch-Meteorologisches Observatorium Davos/World Radiation Center & Switzerland & PMOD/WRC & (Reference) \\
\hline National Oceanic and Atmospheric Administration, Central UV Calibration Facility & USA & CUCF & YES 000904 \\
\hline Innsbruck Medical University, Division for Biomedical Physics & Austria & UIIMP & Scintec 349 \\
\hline Aristotle University of Thessaloniki, Laboratory of Atmospheric Physics & Greece & LAP & YES 921116 \\
\hline Instituto Nacional de Técnica Aerospacial & Spain & INTA & YES 990608 \\
\hline STUK, Radiation and Nuclear Safety Authority, Finland & Finland & STUK & SL $635 \mathrm{D}$ \\
\hline Norwegian Radiation Protection Authority & Norway & NRPA & SL $616 \mathrm{D}$ \\
\hline
\end{tabular}

cross-comparison between the institutes using PMOD/WRC as transfer standard.

It is the first time that such a large-scale intercomparison of UV calibration facilities has been performed. The results of this study show the level of consistency currently achievable in the calibration of broadband UV radiometers measuring erythemally weighted UV radiation by different laboratories. This effort fits within the declared goal of the WMO-GAW strategic plan 2008-2015 to link UV calibration services in different regions (Müller et al., 2007).

\section{Methods}

UV broadband radiometers are designed for measuring the incoming irradiance weighted with a specific spectral responsivity, e.g. the action spectrum for ultraviolet induced erythema (McKinlay and Diffey, 1987; ISO, 1999). The output signal of these instruments depends therefore on the intensity of the receiving radiation and on its spectral shape. The knowledge about the detector spectral responsivity is an important step in the calibration procedure. As this function differs from the nominal action spectrum, a suitable conversion is required to convert from the detector weighted radiation to the one representative for the desired weighting.

A second requirement for such instruments is the weighting of the radiation with the cosine of the incoming angle relative to normal incidence. This ideal case can be fulfilled only to a certain degree by the input optics of the detector. In the UV wavelength range the resulting deviation depends strongly on the solar zenith angle and also on the atmospheric situation, because the ratio of the direct unscattered solar radiation to the diffuse radiation changes considerably during the day.

To account for the intrinsic properties of broadband detectors the calibration procedure includes three steps. First, the spectral response function (SRF) is determined. Second, the angular response function (ARF) is measured in the laboratory. Third, the absolute calibration factor of the radiometer is derived from a direct comparison to a reference instrument. This calibration method is described in Hülsen and Gröbner (2007).

\subsection{The COST726 campaign}

During the PMOD/WRC-COST726 characterisation and calibration campaign (Gröbner et al., 2007), a total of 36 UV broadband radiometers where calibrated at PMOD/WRC, from 30 July to 25 August 2006.

Six of these detectors belong to UVCFs as listed in Table 1 . These radiometers were characterized and calibrated at their home institute prior to the COST726 campaign. This allowed first the intercomparison of the laboratory measurements (SRF and ARF) and secondly to compare the absolute calibration factors of the instruments. The unprocessed (raw) data of the instruments, obtained during the outdoor calibration period at PMOD/WRC, were sent to the respective home institutes. There the raw data were converted to erythemally weighted irradiances using the owners specific conversion procedures. From this processed data a "blind" intercomparison relative to the PMOD/WRC calibration was performed.

\subsection{Laboratory characterization}

The relative spectral response facility in use at the seven UVCFs is quite similar and essentially consists of a single or double monochromator which produces a nearly monochromatic beam of radiation which irradiates the radiometer. The spectral responsivity of the radiometer is retrieved by adjusting the monochromator to successive wavelengths between about 270 and $400 \mathrm{~nm}$. The width of the monochromator output slit function is a compromise between the output intensity and the wavelength resolution of the system $(1.9,0.75,4$, 9, 2.1, 2 and $1.6 \mathrm{~nm}$ for PMOD/WRC, CUCF, UIIMP, LAP, INTA, STUK and NRPA respectively).

For the measurement of the angular response function the radiometer is mounted on a goniometer. The detector sensor is illuminated by a radiation source which is mounted at a distance of at least $1 \mathrm{~m}$ from the goniometer. Either a high intensity Xenon or tungsten-halogen lamp is used. 


\subsection{Absolute calibration}

When the radiometer is used for measuring erythemally weighted solar irradiance, the best radiation source for the absolute calibration is the sun, because the detector output signal depends significantly on the spectral shape of the receiving radiation.

The instrument of choice for the measurement of absolute spectral solar radiation is a well characterized spectroradiometer which is installed in close proximity to the broadband radiometer. At PMOD/WRC the spectroradiometer QASUME is used as the reference instrument, which represents the European reference for spectral solar UV irradiance (Gröbner et al., 2005, 2006; Gröbner and Sperfeld, 2005).

During the outdoor calibration period the reference and the broadband instruments measure simultaneously the solar radiation continuously for several days. From this dataset the sensitivity of the radiometer is retrieved following a calibration procedure outlined in the following section.

\subsection{Determination of the calibration factors and functions}

The first step of the calibration is the determination of a conversion function, $f$, to convert the detector weighted solar irradiance to erythemally weighted irradiance. It is defined as:

$f\left(\mathrm{SZA}, \mathrm{TO}_{3}\right)=\frac{\int \mathrm{CIE}(\lambda) E_{\mathrm{rad}}\left(\mathrm{SZA}, \mathrm{TO}_{3}, \lambda\right) d \lambda}{\int \mathrm{SRF}(\lambda) E_{\mathrm{rad}}\left(\mathrm{SZA}, \mathrm{TO}_{3}, \lambda\right) d \lambda}$,

where $E_{\text {rad }}$ represents solar spectra calculated with a radiative transfer model for different solar zenith angles (SZA) and total ozone column $\left(\mathrm{TO}_{3}\right)$ (Lantz et al., 1999; Leszczynski et al., 1998). The SRF is obtained from the laboratory measurement described in Sect. 2.2 and CIE represents the erythemal action spectrum (McKinlay and Diffey, 1987; ISO, 1999).

Most UVCFs use the libradtran package (Mayer and Kylling, 2005) or similar models to simulate the solar spectrum. The input parameters vary depending on the actual installation place of the radiometer. However, the variation of these parameters have only an effect smaller than $1 \%$ on the variability of $f$ (Hülsen and Gröbner, 2007).

Any deviations of the angular response of the detector entrance optic from the nominal cosine response will result in systematic measurement errors depending on the current atmospheric conditions. This error is usually called cosine error and can be partially corrected using the methodology described in Gröbner et al. (1996); Bais et al. (1998).

The cosine error of an instrument depends on the radiance distribution of the incident radiation which is usually separated into the direct and diffuse radiation component, $E_{\text {dir }}$ and $E_{\text {dif }}$. The standard procedure to correct for a detector cosine error is based on the following equations:

Coscor $=\frac{1}{f_{\text {glo }}}$, $f_{\mathrm{glo}}=f_{\mathrm{dir}} \frac{E_{\mathrm{dir}}}{E_{\mathrm{glo}}}+f_{\mathrm{dif}} \frac{E_{\mathrm{dif}}}{E_{\mathrm{glo}}}$,

where $f_{\text {glo }}$ is the global cosine error and $E_{\text {glo }}$ is the sum of $E_{\text {dir }}$ and $E_{\text {dif }} ; f_{\text {dir }}$ represents the direct cosine error which is equal to the ARF obtained in the laboratory divided by the cosine of the zenith angle and $f_{\text {dif }}$ is called the diffuse cosine error and is here calculated by assuming a homogeneous radiance distribution integrated over the whole hemisphere,

$f_{\text {dif }}=2 \cdot \int_{0}^{\frac{\pi}{2}} \operatorname{ARF}(\Theta) \sin (\Theta) d \Theta$.

The direct and diffuse radiation components $E_{\text {dir }}$ and $E_{\text {dif }}$ are usually estimated by radiative transfer calculations as done by CUCF, INTA, NRPA and PMOD/WRC. Another approach is to implicitly include an average cosine error of the radiometer into its absolute calibration by retrieving an absolute calibration as a function of SZA. This is the method used by LAP, UIIMP and STUK.

To calculate the erythema weighted irradiance from the raw data of a broadband radiometer the following equation is used (Webb et al., 2006):

$E_{\mathrm{CIE}}=\left(U-U_{\mathrm{offset}}\right) \cdot C \cdot f_{\mathrm{n}}\left(\mathrm{SZA}, \mathrm{TO}_{3}\right) \cdot$ Coscor,

where $U$ and $U_{\text {offset }}$ are the raw and dark signal respectively and $C$ represents the absolute calibration factor. The conversion function $f_{\mathrm{n}}$ is calculated according to Eq. (1) and is normalized to its value $f_{0}$ at $\mathrm{SZA}=40^{\circ}$ and $\mathrm{TO}_{3}=300 \mathrm{DU}$. If the cosine error of the instrument is explicitly taken into account, it is corrected by the Coscor-function (Eq. 2, CUCF, INTA, NRPA, PMOD/WRC), otherwise it is set to unity (LAP, UIIMP, STUK).

The dark signal $U_{\text {offset }}$ is obtained from the average of a large number of nighttime readings of the radiometer. The calibration factor $C$ is calculated for each solar irradiance scan by the comparison of the SRF-weighted solar spectrum measurement $E_{\mathrm{D}}$ with the representative radiometer signal $U_{\mathrm{D}}$ :

$C=\frac{E_{D}}{U_{D}-U_{\text {offset }}} \cdot \frac{1}{\text { Coscor }} \cdot f_{0}$,

where $f_{0}$ is the normalization factor of the conversion function. $U_{\mathrm{D}}$ is obtained from a judicious combination of the individual radiometer signals during the solar spectrum scan (see Hülsen and Gröbner, 2007, for the PMOD/WRC method; methods from other institutes differ slightly). The retrieved calibration factor $C$ should be the same under all atmospheric conditions and for all radiation spectra. If any significant variability of $C$ is observed (for example depending on SZA) this would indicate a mismatch of the measured SRF and ARF with the radiometer characteristics at the time of the solar measurements, or an inadequate cosine correction. If no cosine correction is available (Coscor=1), the absolute calibration factor becomes a function depending on SZA and possibly also on other factors. 
The final absolute calibration factor is obtained as the average of all measurements satisfying a pre-defined set of criteria, e.g. at this campaign for measurement conditions without precipitation and SZA smaller than $75^{\circ}$.

\subsection{Deviations from Eq. (5)}

- CUCF: the calibration is performed not for a single radiometer relative to the reference instrument but for a radiometer triad. The absolute calibration factor is therefore the mean of the triad and an additional scaling factor is needed.

- UIIMP, LAP and INTA: the absolute calibration factor $C$ obtained from the clear sky calibration periods is included in the conversion function $f_{n}$ as a function $C^{\prime}$ (SZA).

- STUK: a single absolute calibration factor is used to convert the raw data to erythema weighted irradiance $\left(\right.$ Coscor $\left.=1, f_{n}=1\right)$. The normalization factor of the conversion function, $f_{0}$, and the cosine error are implicitly integrated in $C$ during the outdoor calibration.

\section{Results}

The intercomparison of the UVCFs was accomplished through bilateral comparisons between calibrations established by the UVCFs and PMOD/WRC. Thus, even though there were no direct comparisons between the UVCFs, PMOD/WRC acted as the pilot laboratory and through its performance all UVCFs can be related to each other. In the following section, the outdoor measurements of each radiometer, processed by the respective UVCF, will be compared to the PMOD/WRC processed data and analysed with respect to the laboratory characterisations done at both laboratories. Thus, the consistency of the whole calibration chain of a UV broadband radiometer will be investigated and discussed.

\subsection{Laboratory characterization}

\subsubsection{Spectral response function}

The spectral response functions of the UV broadband radiometers investigated in this study span about 3 orders of magnitude over a $40 \mathrm{~nm}$ region, between their maximum at about $297 \mathrm{~nm}$ to the low sensitivity plateau starting at around $340 \mathrm{~nm}$, similarly to the definition of the CIE erythemal action spectrum. Potential errors in the wavelength calibration and the determination of the spectral transmission function of the monochromatic source may introduce significant discrepancies in the derived spectral response function of the test radiometers.

Figure 1 shows the SRF as derived by PMOD/WRC and the other UVCFs for each radiometer. The ratio of the two data sets is presented in the corresponding lower figures. The agreement between the measurements is fairly consistent in the shorter wavelength range, up to about $340 \mathrm{~nm}$, with deviations not exceeding $\pm 20 \%$ for most institutes. Larger deviations are only found for two institutes. The large sensitivity gradient between about 300 and $340 \mathrm{~nm}$ is reproduced faithfully by all institutes. Measurements in that wavelength range are strongly influenced by the resolutions of the respective monochromatic sources, and observed deviations between institutes, such as between UIIMP and PMOD/WRC for example, can be explained by this effect (Schreder et al., 2004).

At wavelengths longer than approx. $340 \mathrm{~nm}$ the measurement of the SRF becomes difficult due to the low signal of the radiometer and the correspondingly high noise level of the measurements. This is the reason for the limited extent of the SRF measurements for some radiometers, particularly the YES UVB-1 radiometers which have an unusually high noise level which limit the SRF measurement to about $340 \mathrm{~nm}$. However, improvements to the spectral response bench at CUCF have allowed better measurements in the tail region of the SRF of the YES UVB 000904 (Fig. 1a). For the Solar Light 501 digital radiometers the limitation comes from the low resolution of the digital recorders manufactured by Solar Light. This can be overcome by sampling the output signal by a custom made readout electronic, as was done at STUK (Fig. 1e). The SRF of the Solar Light 616 from NRPA could be obtained at PMOD/WRC and NRPA with a good agreement (Fig. 1f); nevertheless the SRF measurement performed at PMOD/WRC shows slightly higher noise in the UVA range which could be improved by increasing the sampling time at each wavelength step.

\subsubsection{Angular response}

Figure 2 shows the cosine errors derived from the measured ARF's. The differences between the measurement performed at PMOD/WRC and the other UVCFs is below $\pm 4 \%$ for zenith angle less than $75^{\circ}$. This result shows that the angular response can be measured with high accuracy by different laboratories.

\subsubsection{Derived conversion and cosine correction functions}

Figure 3 shows the conversion functions $f$ as derived from the SRF measurements (Fig. 1) using Eq. (1). For the calculation missing data of the SRF must be extrapolated to fill the full UV wavelength range. Either a linear or logarithmic extrapolation from the last measured point to the point $\operatorname{SRF}(400 \mathrm{~nm}) \approx 10^{-6}$ was used to complete the dataset, or the missing data points were set to a fixed value (zero or $\approx 10^{-6}$ ). But although each institute used a different extrapolation, the resulting conversion functions are nearly identical. The good agreement of $f$ between the institutes and PMOD/WRC also underlines the fact that the choice of parameters to calculate 

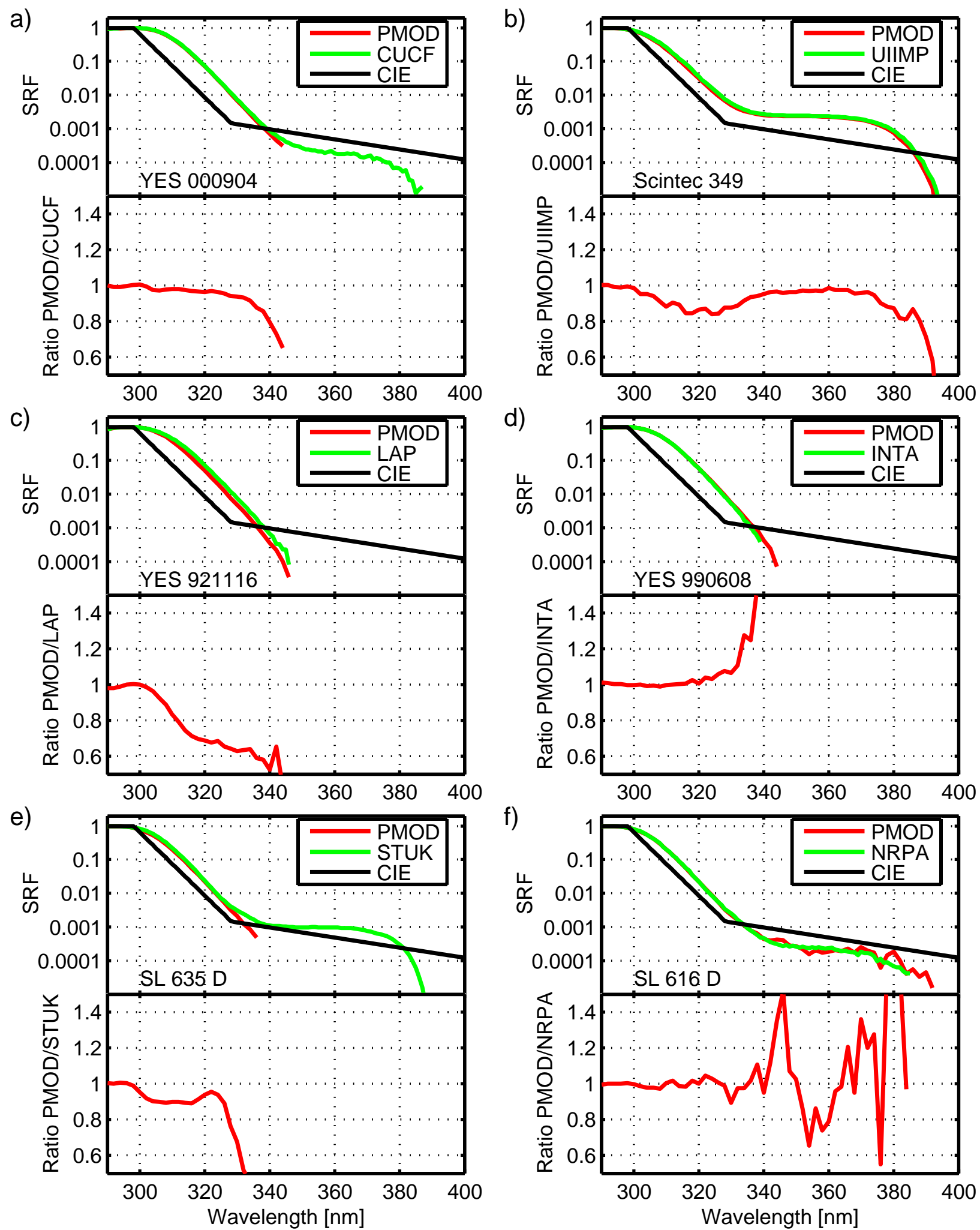

Fig. 1. Spectral response functions as measured at PMOD/WRC and at the owners calibration facility (see Table 1). The ratio of the two measurements are shown in the bottom half of the respective figure. 
a)

c)

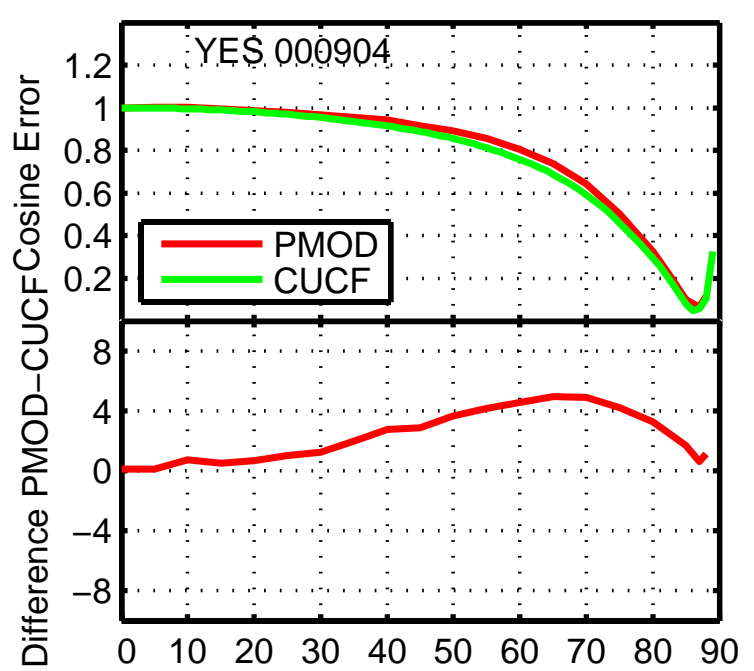

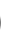

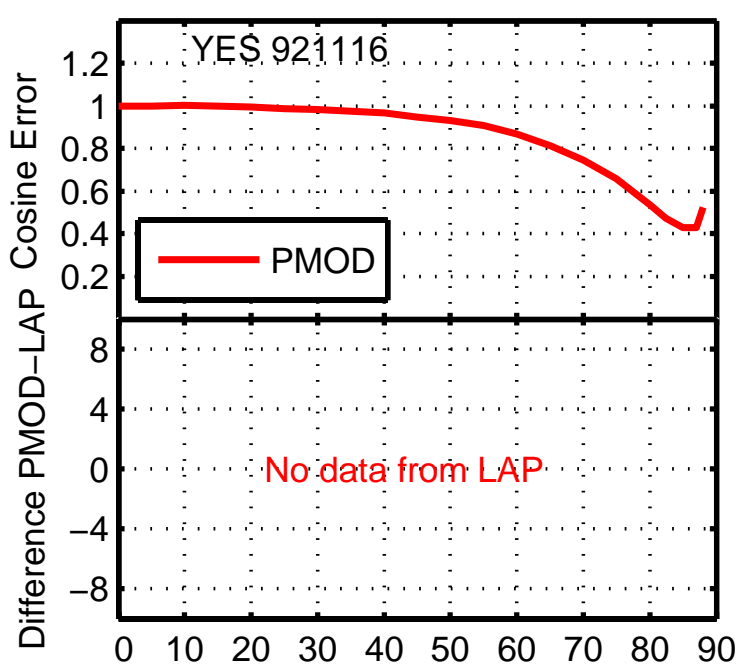

e)

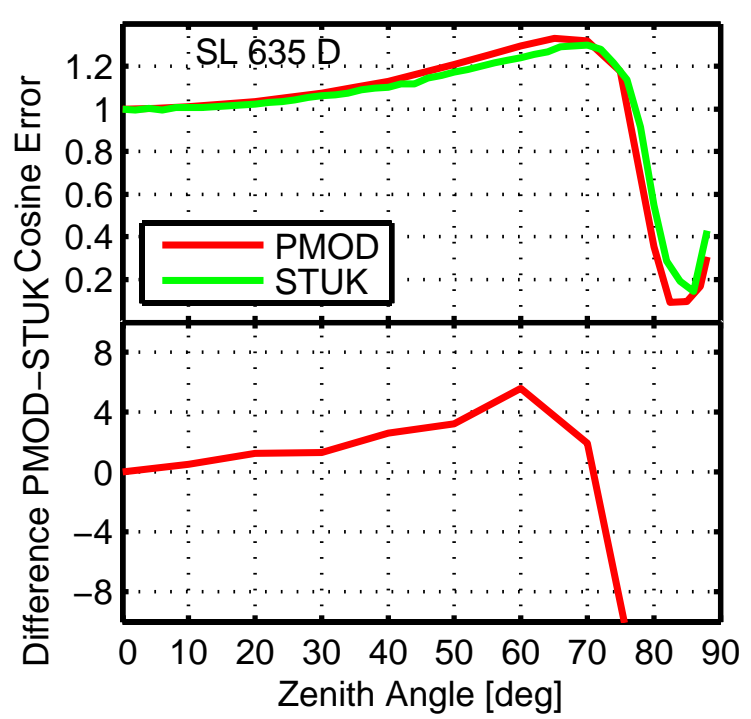

b)

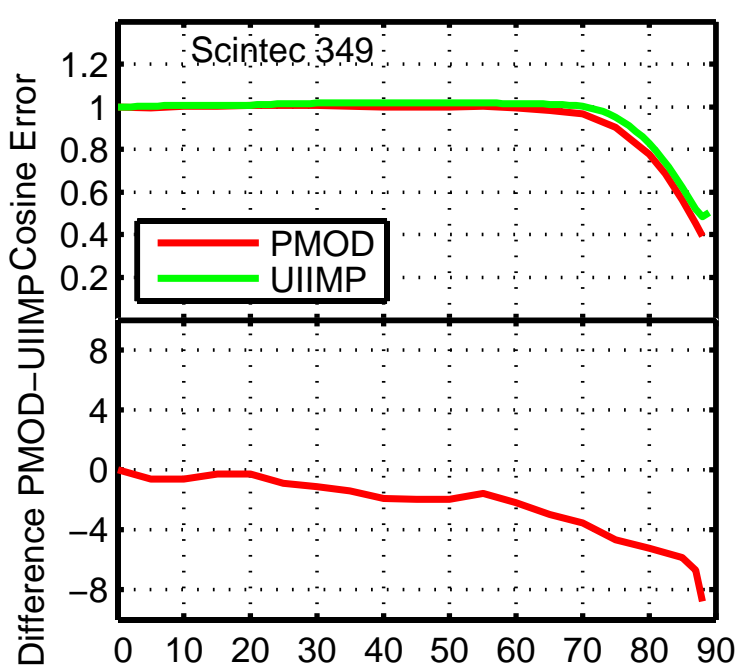

d)

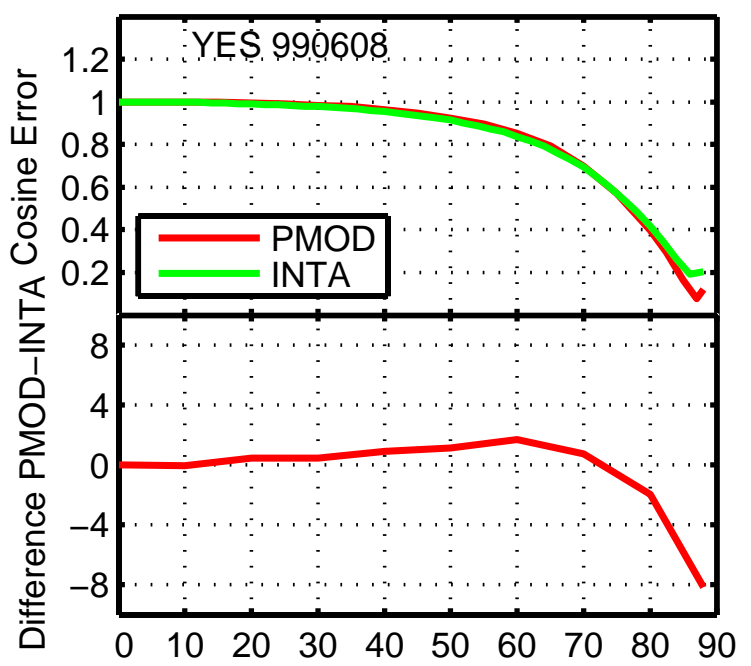

f)

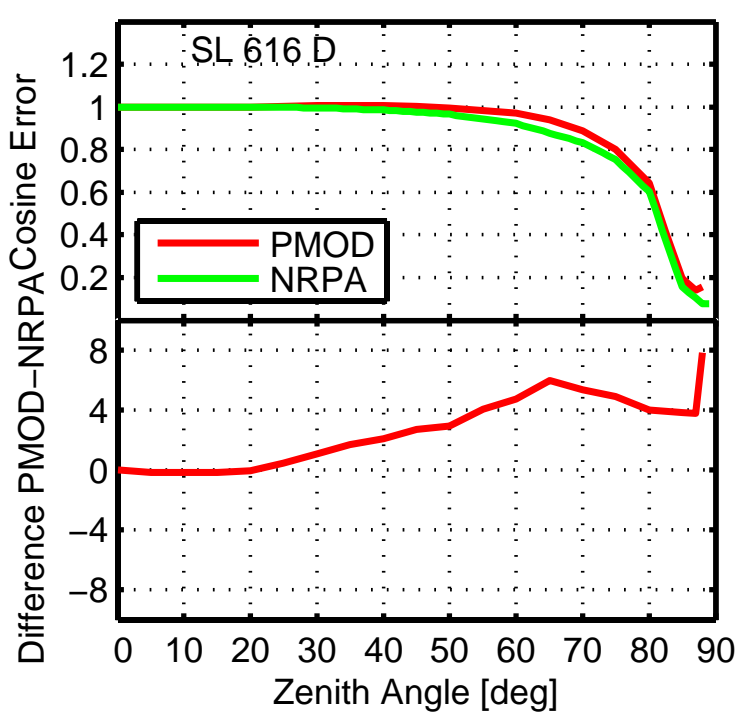

Fig. 2. Cosine Error derived from the angular response functions as measured at PMOD/WRC and at the owners calibration facility (see Table 1). The difference of the two measurements in percent are shown in the bottom half of each figure. The ARF of the YES 921116 radiometer was not determined at LAP before the COST726 campaign. 
a)

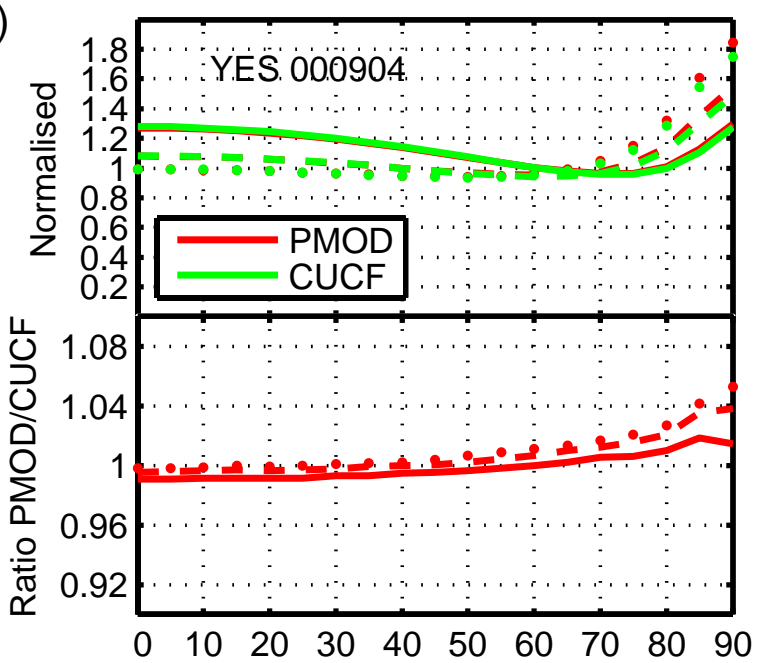

c)

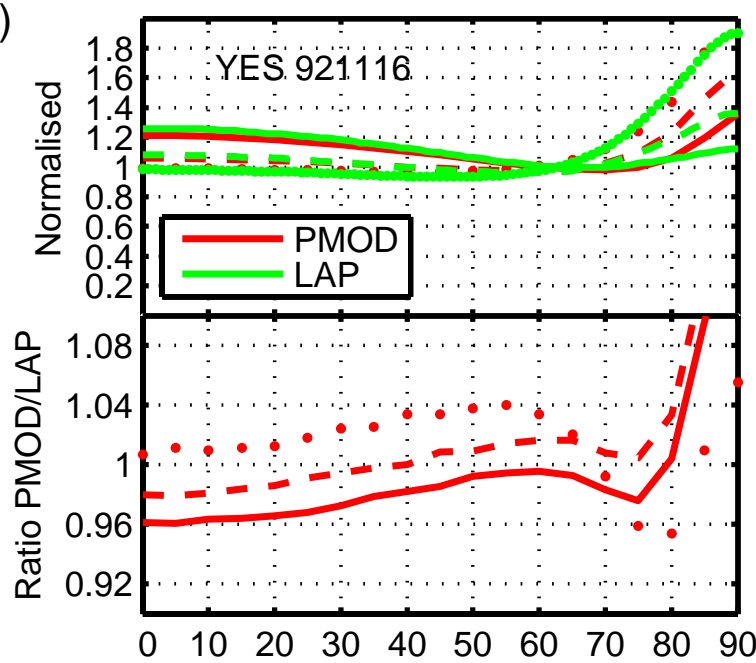

e)

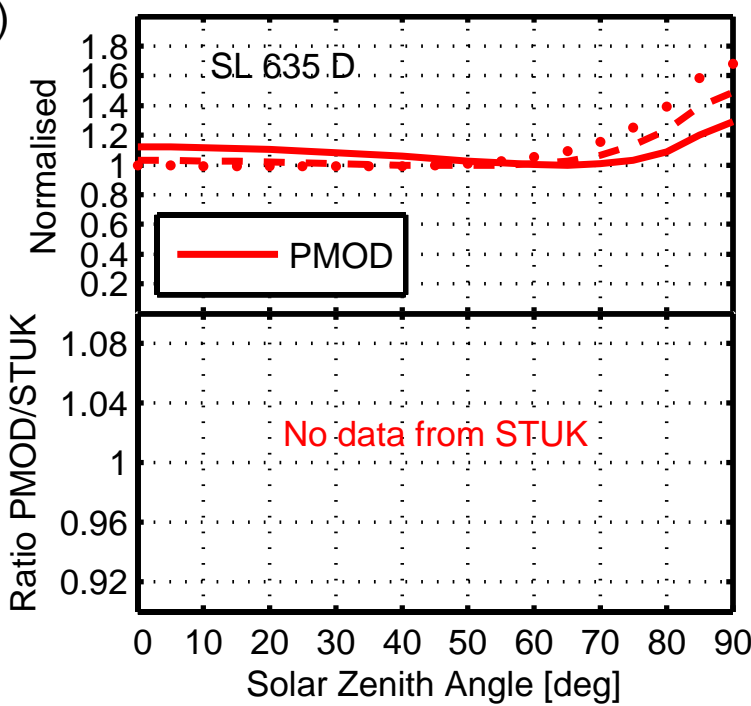

b)

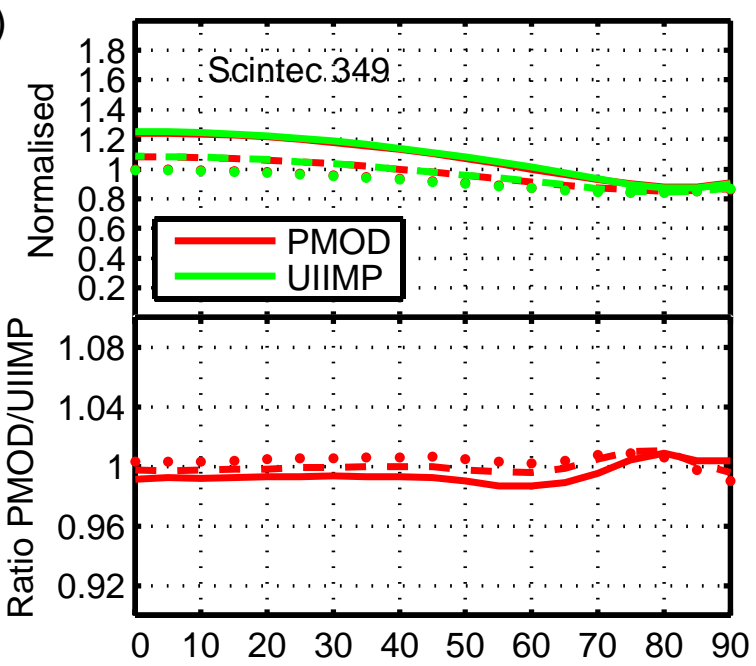

d)

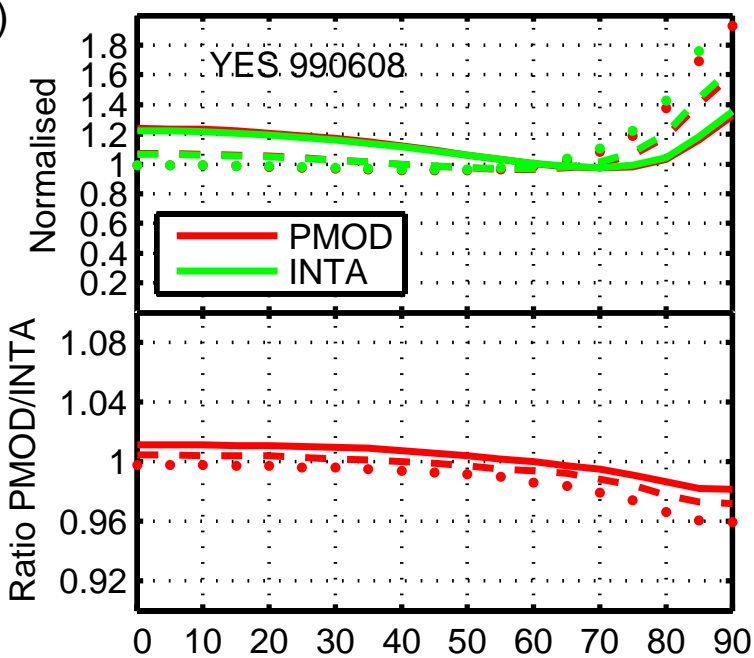

f)

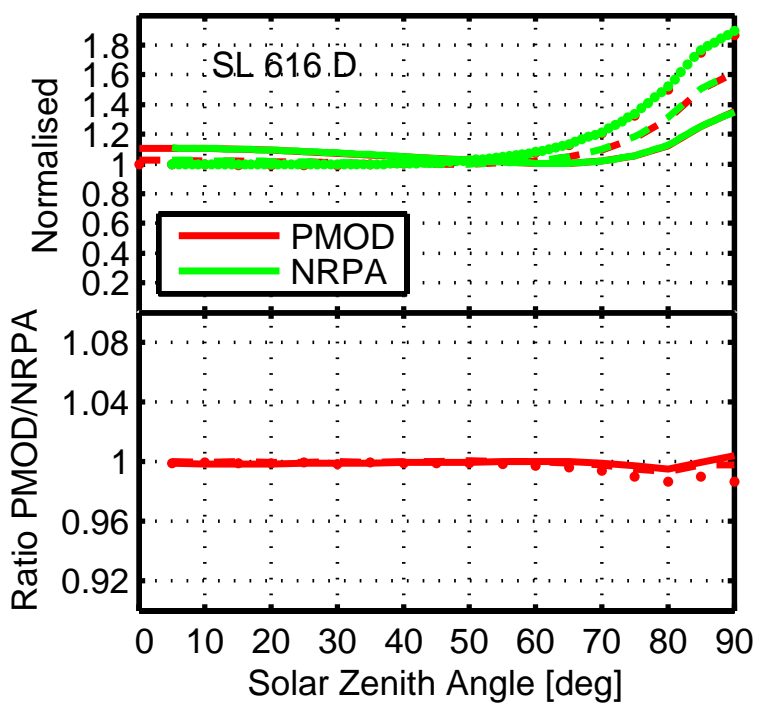

Fig. 3. Conversion function $f$ in dependence of solar zenith angle for $200 \mathrm{DU}$ (solid line), $300 \mathrm{DU}$ (dashed line) and $400 \mathrm{DU}$ (dotted line) calculated using the SRF measured respectively by PMOD/WRC and the home institute (see Fig. 1). The ratio of the two conversion functions are shown in the bottom half of each figure. The conversion function of the Solar Light 635 radiometer was not determined at STUK. 
Table 2. Diffuse cosine error calculated according to Eq. (4) using the measured angular response functions shown in Fig. 2.

\begin{tabular}{lllc}
\hline Instrument & PMOD/WRC & Owner & PMOD/Owner [\%] \\
\hline YES 000904 & 0.85 & 0.82 & +3 \\
Scintec 349 & 0.98 & none & none \\
YES 921116 & 0.90 & none & none \\
YES 990608 & 0.88 & 0.87 & +1 \\
SL 635 D & 1.12 & 1.10 & +2 \\
SL 616 D & 0.95 & 0.93 & +2 \\
\hline
\end{tabular}

Table 3. Clear sky cosine correction factor at $\mathrm{SZA}=40^{\circ}$ calculated according to Eq. (2) using the measured angular response functions shown in Fig. 2.

\begin{tabular}{llll}
\hline Instrument & PMOD/WRC & Owner & PMOD/Owner [\%] \\
\hline YES 000904 & 1.121 & 1.157 & -3.1 \\
Scintec 349 & 1.010 & none & none \\
YES 921116 & 1.075 & none & none \\
YES 990608 & 1.088 & 1.100 & -1.1 \\
SL 635 D & 0.889 & none & none \\
SL 616 D & 1.021 & 1.048 & -2.6 \\
\hline
\end{tabular}

the spectra ( $E_{\text {rad }}$ in Eq. 1) used to derive $f$ do not introduce any significant discrepancies in the determination of $f$.

For most conversion functions the ratio between PMOD/WRC and the owners calculation are within $\pm 2 \%$. The observed differences in the SRF measurements, as discussed in the previous section are therefore not significant. This is not the case for the conversion function of the YES 921116 from LAP where a significant difference with the PMOD/WRC can be seen (Figure 3c). The deviations exceed $\pm 4 \%$ for higher SZA and the functions differ by more than $5 \%$ for $\mathrm{TO}_{3}$ values between 200 and $400 \mathrm{DU}$. These differences were traced to the different determinations of the respective SRF measurements of both institutes as could be verified by using the same radiative transfer model spectra to derive $f$.

The cosine correction functions for nominal diffuse and clear sky were derived from the angular response functions (Fig. 2) using Eqs. (2) to (4). The diffuse and clear sky cosine errors are shown in, respectively, Table 2 and Table 3 . The differences between the owners institutes and PMOD/WRC are usually below $\pm 3 \%$, which indicates that the methods used to derive the cosine correction functions from the ARF measurements were consistent between all institutes.

\subsection{Absolute calibration factor}

The absolute calibration factors $C$ derived from the outdoor measurement campaign are given in Table 4. As some insti-
Table 4. Absolute calibration factor at $\mathrm{TO}_{3}=300 \mathrm{DU}$ and $\mathrm{SZA}=40^{\circ}$. For comparibility, the owners calibration factors indicated with $*$ are divided by the clear sky cosine correction calculated by PMOD/WRC since these institutes do not separate the absolute calibration factor and the cosine correction. The units are in $\mathrm{W} \mathrm{m}^{-2} / \mathrm{V}$ for the first four radiometers and in $\mathrm{W} \mathrm{m}^{-2} / \mathrm{MED} \mathrm{h}^{-1}$ for the last two (Solar Light).

\begin{tabular}{lllc}
\hline Instrument & PMOD/WRC & Owner & PMOD/Owner [\%] \\
\hline YES 000904 & 0.1151 & 0.1126 & +2.2 \\
Scintec 349 & 0.1480 & $0.1524^{*}$ & -3.0 \\
YES 921116 & 0.1506 & $0.1570^{*}$ & -4.1 \\
YES 990608 & 0.1199 & 0.1183 & +1.4 \\
SL 635 D & 0.0569 & $0.0525^{*}$ & +8.4 \\
SL 616 D & 0.0559 & 0.0549 & +1.9 \\
\hline
\end{tabular}

tutes (UIIMP, LAP and STUK) did not explicitly correct $C$ with the cosine error of their radiometer (Eq. 5), the comparison of these derived calibration factors are affected by the cosine errors of the radiometers. So as to provide a meaningful comparison in these cases, the listed values of $C$ correspond to the absolute calibration factors corrected by the clear sky cosine correction factor derived by PMOD/WRC. It should be noted that this procedure introduces systematic differences in the derivation of $C$ due to the differences between the theoretical clear sky cosine correction and the average cosine error of the radiometer which will depend on the atmospheric conditions during the calibration period at the respective UVCFs. Nevertheless a good agreement of the order of $\pm 4 \%$ could be found between the majority of calibration facilities which is very satisfying considering the difficulties in measuring accurately global spectral solar irradiance.

The deviation of approx. $4 \%$ found between LAP and PMOD/WRC can be attributed to differences in the absolute calibrations of the reference spectroradiometers used to measure the reference solar spectra. This was verified during a QASUME quality assurance site audit in 2002 were a mean spectral difference of $3.8 \%$ between the spectrophotometer of LAP (Brewer \#086 - GRT) and QASUME was found (Gröbner et al., 2003). In the case of STUK, the large deviation of approx. $8 \%$ is so far unexplained.

\subsection{Intercomparison of erythemally weighted irradiances}

The calibration factors and correction functions introduced previously were used to convert the raw data of the radiometers to erythemally weighted irradiance using Eq. (5) (or the corresponding equation used by the respective UVCF). The raw data was sent to each UVCF to be processed using their own calibration procedures; the processed data was then forwared to PMOD/WRC which performed the comparison with the PMOD/WRC derived values. This intercomparison 
a)

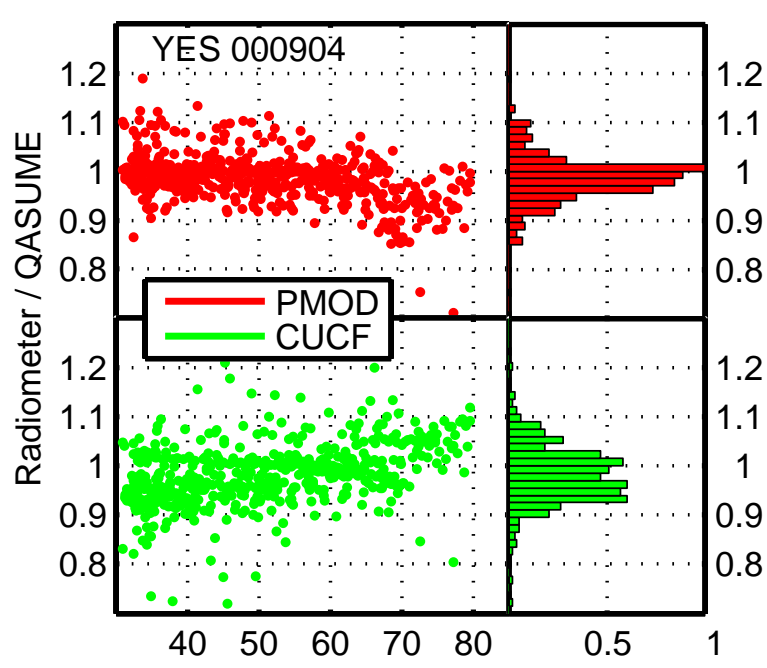

c)

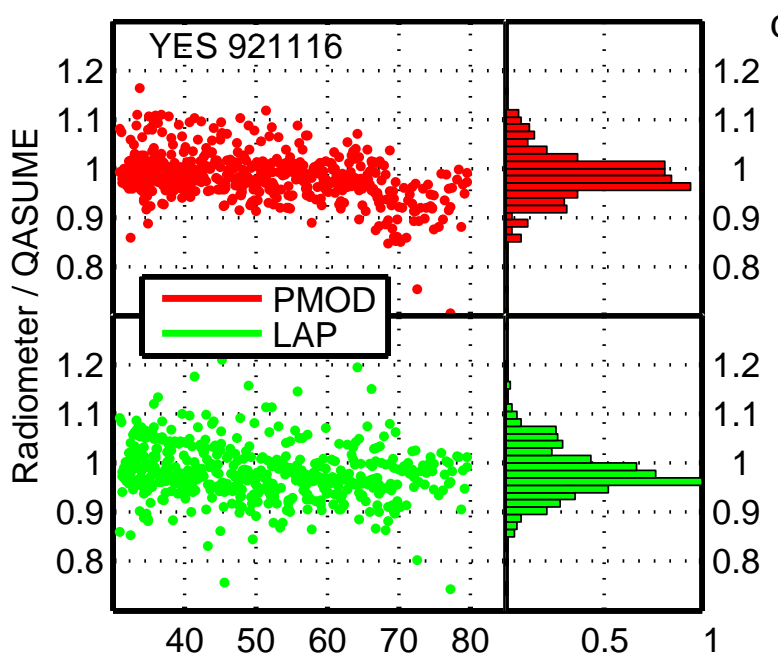

e)

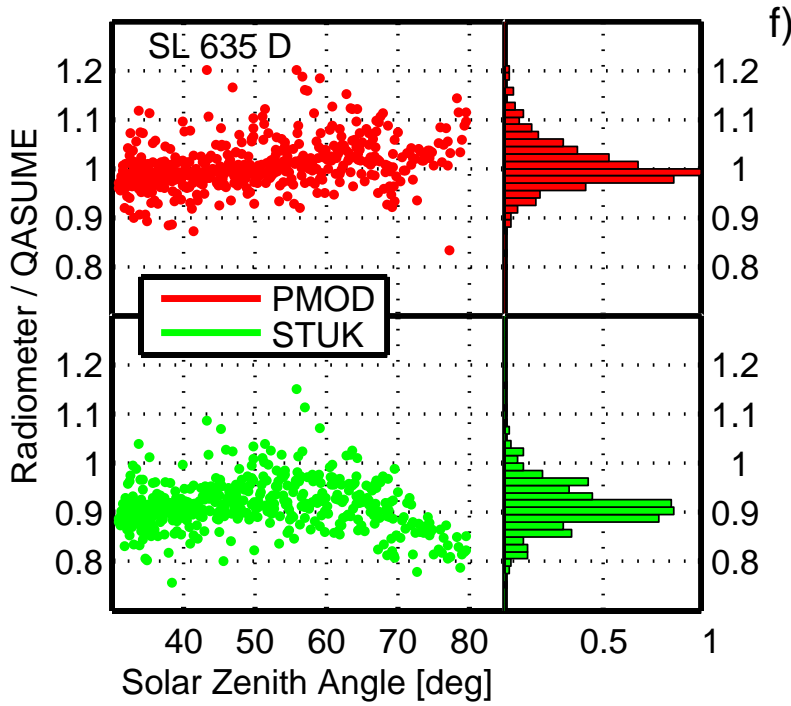

b)

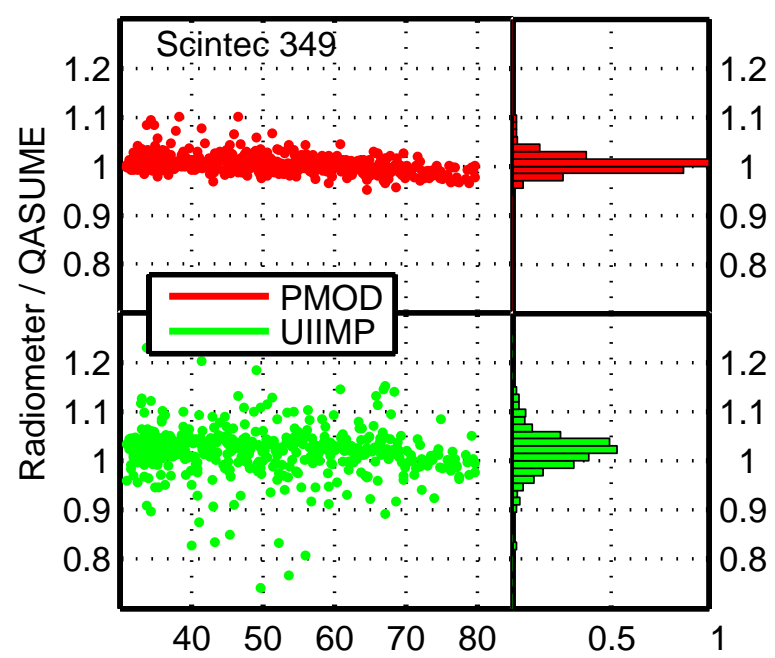

d)
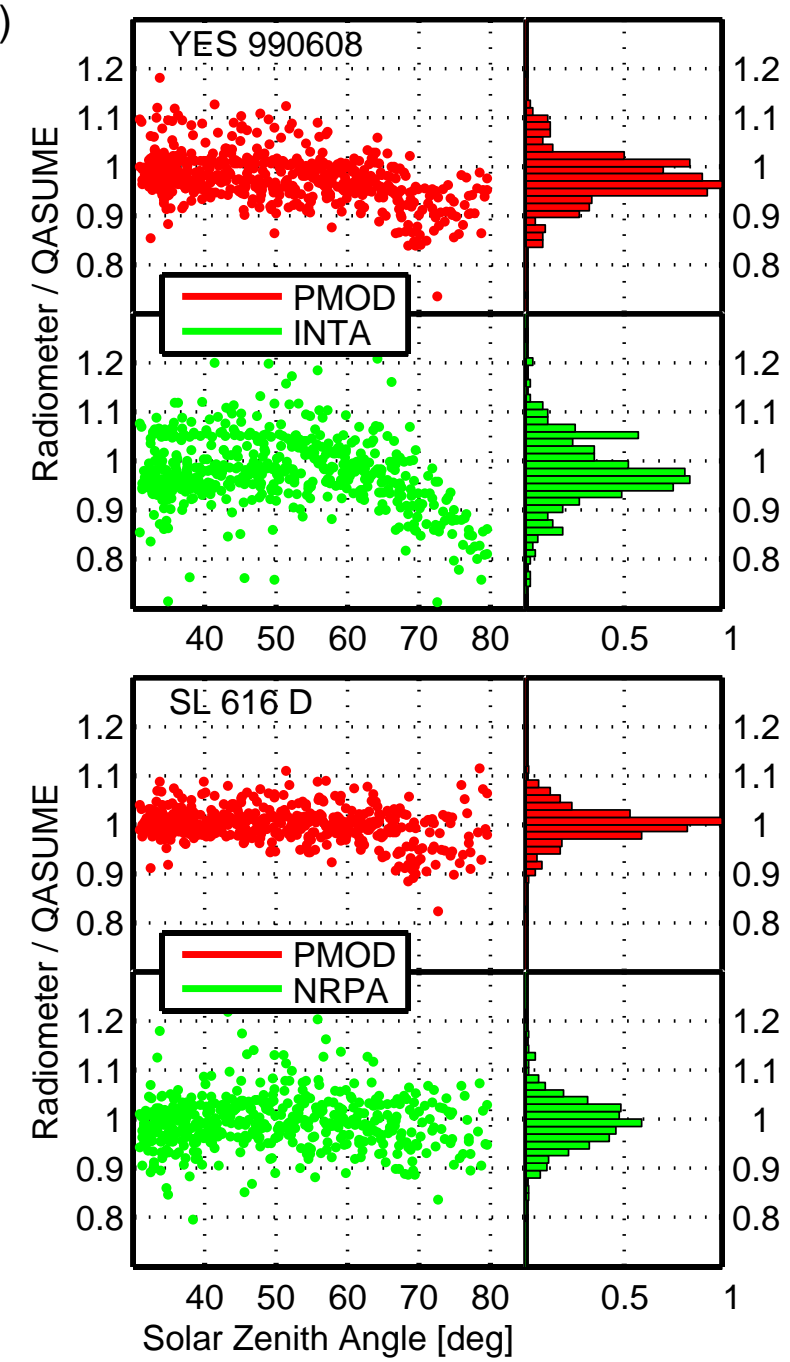

Fig. 4. Erythemally weighted irradiances derived by the PMOD/WRC and the respective home institute relative to the QASUME spectroradiometer for the whole measurement campaign in dependence on the solar zenith angle. The right side of each figure shows the corresponding residuals in bins of 0.015 . The histograms are normalized to the largest bin in each figure. 
Table 5. Summary results of the outdoor measurement campaign (see also Fig. 4). The second and third columns list the mean and standard deviation of the erythemally weighted irradiances ratios between the radiometer and the QASUME reference spectroradiometer, calibrated by PMOD/WRC and the owners, respectively.

\begin{tabular}{lll}
\hline Instrument & PMOD/WRC & Owner \\
\hline YES 000904 & $0.985 \pm 0.049$ & $0.982 \pm 0.063$ \\
Scintec 349 & $1.004 \pm 0.019$ & $1.020 \pm 0.054$ \\
YES 921116 & $0.983 \pm 0.050$ & $0.981 \pm 0.061$ \\
YES 990608 & $0.975 \pm 0.052$ & $0.977 \pm 0.074$ \\
SL 635 D & $1.006 \pm 0.049$ & $0.912 \pm 0.051$ \\
SL 616 D & $1.000 \pm 0.035$ & $0.990 \pm 0.071$ \\
\hline
\end{tabular}

was "blind" in the sense that no information was exchanged between the institutes prior to the comparison performed by PMOD/WRC. Any later submission of newly processed data was labeled as revised and required a detailed explanation by the corresponding institute. Only LAP submitted a revised data set due to the discovery of a software error in their processing chain (Gröbner et al., 2007, p. 100-103).

The results are summarized in Fig. 4 and the mean ratios to the reference spectroradiometer QASUME are listed in Table 5 . These final results show that the erythemally weighted irradiances derived by the majority of UVCFs are consistent to within $\pm 2 \%$. The variability between the radiometers and the QASUME reference spectroradiometer can be largely attributed to the challenging meteorological conditions of the campaign, which consisted of only one and a half clear sky days, while the most part of the campaign was either fully overcast or with rapidly changing cloud conditions. The latter introduced a large variability in these radiometers having a large cosine error since these days were treated as diffuse in terms of the applied cosine correction even though clear sky periods (solar disk free of clouds) alternated with overcast conditions. As discussed in Hülsen and Gröbner (2007) this can lead to variabilities of up to $\pm 7.2 \%$ for radiometers with a large cosine error such as the YES radiometers. This is confirmed by the lower variabilities of the Scintec radiometer which has a very low cosine error compared to the other radiometers in this study.

Neglecting the cosine correction in Eq. (5) leads to a significant variability in dependence on the SZA for radiometers with a large cosine error as can be seen for the radiometers of INTA and STUK. Neither institute applies a cosine correction and especially at high SZA deviations relative to QASUME of up to $20 \%$ are observed. In the case of STUK the high deviations at high SZA could also be due to the setting of the conversion function $f_{n}$ to unity.

\section{Conclusions}

A joint intercomparison of broadband radiometers measuring erythemally weighted solar irradiance was performed between six UV calibration facilities in Europe and one in the USA. The characterisation and calibration campaign was organised by PMOD/WRC in Davos, Switzerland.

The owners calibrated their UV broadband radiometers prior to sending them to PMOD/WRC. The subsequent calibration done by PMOD/WRC was compared to the owners calibration. It was assumed that the radiometers did not significantly change from the time of the calibration performed at the home insitute and the one done at PMOD/WRC.

The calibration consisted in the determination of the spectral and angular response of the radiometer, followed by an absolute calibration performed outdoors relative to a spectroradiometer which provided the absolute reference.

The characterization of the detectors in the respective laboratories were found to be in good agreement, especially concerning the determination of the angular response, with deviations below $\pm 4 \%$ in the calculated cosine error. The larger differences observed with the spectral response functions is due to the differences in the laboratory setups used to determine the SRF. However the differences do not introduce any significant discrepancies in the resulting calibration apart from one case.

A "blind" intercomparison of the erythemally weighted irradiances derived by the respective institutes and PMOD/WRC showed consistent measurements to within $\pm 2 \%$ for the majority of institutes. Only one institute (STUK) showed slightly larger deviation of $10 \%$ (see Table 5 and Fig. 4).

The absolute calibration of the spectroradiometers, which are used to calibrate the erythema detectors, has an uncertainty of at least $\pm 5 \%$. Therefore the results of the intercomparison are very good, since nearly all instrument calibrations are well within their estimated uncertainties.

Acknowledgements. The instrumentation of the UV Center at PMOD/WRC is made available by the Joint Research Centre of the European Commission in Ispra under the cooperation agreement 2004-SOCP-22187. G. Hülsen acknowledges support from the European Cooperation in the field of Scientific and Technical Research (COST), SBF No. C05.0068. Many thanks also to C. Wilson from CUCF for laboratory measurements of the YES UVB-1 000904.

Edited by: J. Burrows

\section{References}

Bais, A., Kazadzis, S., Balis, D., Zerefos, C., and Blumthaler, M.: Correcting global solar ultraviolet spectra recorded by a Brewer spectroradiometer for its angular response error, Appl. Optics, 37, 6339-6344, 1998. 
Gröbner, J. and Sperfeld, P.: Direct traceability of the portable QASUME irradiance scale to the primary irradiance standard of the PTB, Metrologia, 42, 134-139, 2005.

Gröbner, J., Blumthaler, M., and Ambach, W.: Experimental investigation of spectral glabal irradiance measurement errors due to a non ideal cosine response, Geophys. Res. Lett., 23, 2493-2496, 1996.

Gröbner, J., Kazadzis, S., Schreder, J., Bais, A. F., Blumthaler, M., Görts, P., Koskela, T., Seckmeyer, G., Webb, A. R., and Wuttkeand, S.: Quality assurance of spectral ultraviolet measurements in Europe through the development of a transportable unit, Doc. EUR 20991 EN (European Commission, 2003), 2003.

Gröbner, J., Schreder, J., Kazadzis, S., Bais, A. F., Blumthaler, M., Görts, P., Tax, R., Koskela, T., Seckmeyer, G., Webb, A. R., and Rembges, D.: Traveling reference spectroradiometer for routine quality assurance of spectral solar ultraviolet irradiance measurements, Appl. Optics, 44, 5321-5331, 2005.

Gröbner, J., Blumthaler, M., Kazadzis, S., Bais, A., Webb, A., Schreder, J., Seckmeyer, G., and Rembges, D.: Quality assurance of spectral solar UV measurements: results from $25 \mathrm{UV}$ monitoring sites in Europe, 2002 to 2004, Metrologia, 43, 6671, 2006.

Gröbner, J., Hülsen, G., Vuilleumier, G. L., Blumthaler, M., Vilaplana, J. M., Walker, D., and Gil, J. E.: Report of the PMOD/WRC-COST Calibration and Intercomparison of Erythemal Radiometers, http://i115srv. vu-wien.ac.at/uv/COST726/COST726_Dateien/Results/ PMOD_WRC_COST726_campaign_2006_R.pdf, 2007.

Hülsen, G. and Gröbner, J.: Characterization and calibration of ultraviolet broadband radiometers measuring erythemally weighted irradiance, Appl. Optics, 46, 5877-5886, 2007.

ISO: Erythema reference action spectrum and standard erythema dose, International Organization for Standardization, ISO/CIE 17166:1999, 1999.
Lantz, K., Disterhoft, P., DeLuisi, J., Early, E., Thompson, A., Slusser, J., and Bigelow, D.: A Methodology for Deriving Clear-Sky Erythema Calibration Factors for UVB Broadband Radiometers of the CUCF, J. Atmos. Ocean. Tech., 16, 17361752, 1999.

Leszczynski, K., Jokela, K., Ylianttila, L., Visuri, R., and Blumthaler, M.: Erythemally weighted radiometers in solar UV monitoring: Results from the WMO/STUK Intercomparison, Photochem. Photobiol., 67, 212-221, 1998.

Mayer, B. and Kylling, A.: The libRadtran software package for radiative transfer calculations - description and examples of use, Atmos. Chem. Phys., 5, 1855-1877, 2005, http://www.atmos-chem-phys.net/5/1855/2005/.

McKinlay, A. and Diffey, B.: A reference action spectrum for ultraviolet induced erythema in human skin, CIE Journal, 6, 17-22, 1987.

Müller, G., Artz, R., Baltensperger, U., Carmichael, G., Dlugokencky, E., Penkett, S., Stähelin, J., Webb, A., Hov, O., Klausen, J., Sturges, B., Barrie, L., Braathen, G., Jalkanen, L., and Nickovic, S.: WMO Global Atmosphere Watch (GAW) Strategic Plan: 2008-2015, World Meteorological Organization WMO/GAW Rep., WMO TD NO. 1384, http://www.wmo.int/ pages/prog/arep/gaw/documents/gaw172_sp_15may.pdf, 2007.

Schreder, J., Gröbner, J., Los, A., and Blumthaler, M.: Intercomparison of monochromatic source facilities for the determination of the relative spectral response of emal broadband filter radiometers, Opt. Lett., 29, 1455-1457, 2004.

Webb, A., Gröbner, J., and Blumthaler, M.: A Practical Guide to Operating Broadband Instruments Measuring Erythemally Weighted Irradiance, http://i115srv.vu-wien.ac.at/uv/COST726/ COST726_Dateien/Results/GuideBB_COST726.pdf, EUR 22595, ISBN 92-898-0032-1, 2006. 